\section{Study of the use of cefovecin (Convenia; Zoetis UK) in cats in first opinion practice in the UK, using the SAVSNET database}

\section{Sara Burke', Angie Hibbert ${ }^{1}$, Vicki Black', Fernando Sánchez-Vizcaíno Buendía², Sarah Whitehouse ${ }^{3}$, Séverine Tasker ${ }^{1}$}

\author{
The Feline Centre, Langford Veterinary Services (LVS), School of \\ Veterinary Sciences, University of Bristol, Bristol, UK \\ University of Liverpool, Institute of Infection and Global Health \\ Leahurst Campus, Neston, Liverpool, UK \\ Institute of Health and Biomedical Innovation, Queensland University \\ of Technology, Brisbane, Queensland, Australia
}

Our objective was to describe the use of cefovecin (Convenia; Zoetis UK), a third generation injectable cephalosporin, in cats in first opinion practice in the UK using the SAVSNET - the Small Animal Veterinary Surveillance Network - database.

Data were obtained as an excel database from SAVSNET for all feline entries containing the word Convenia and/or cefovecin from September 2012-September 2013. Duplicate case entries and those found not to have received Convenia were removed. Using the clinical notes, entries were classified according to body system, microbiological evaluation, concurrent therapies, indications and reason for use. Data were exported into SPSS and descriptive analysis performed. Indications for use were compared to datasheet recommendations.
Of 1489 total entries, 297 duplicate entries and 44 nonConvenia entries were excluded, leaving 1148 entries for analysis. Entries comprised 999 (87\%) non-pedigree, 121 (10.6\%) pedigree and 28 (2.4\%) unknown breeds. There were 574 (50\%) males, 529 (46.1\%) females and 45 (3.9\%) of unknown sex. The median age was 9.1 years, range $0.1-23.2$ years. The median weight was $4.18 \mathrm{~kg}$, range $1.74-9.05 \mathrm{~kg}$.

Indications for Convenia use complied with datasheet recommendations in 739 (64.4\%) entries. Non-datasheet recommendations were described in 306 (26.7\%) entries and 103 (9\%) entries had no indication described. The median dose used was $8.0 \mathrm{mg} / \mathrm{kg}$, range $3.5-21.5 \mathrm{mg} / \mathrm{kg}$. Temperature was recorded in $327(28.5 \%)$ entries; 97 of these $(29.7 \%)$ were $>39^{\circ} \mathrm{C}$

Prior to the use of Convenia, some form of microbiological evaluation (e.g. finding of purulent material, culture and sensitivity [C\&S]) was recorded in 194 (16.9\%) of 1148 entries; only four $(0.35 \%)$ of the 1148 entries had C\&S performed

Of 1148 entries, 525 (45.7\%) had concurrent non-steroidal anti-inflammatory drug treatment, 262 (22.8\%) had concurrent steroid treatment and concurrent antimicrobials were used in 79 (6.9\%) of entries.

A reason for prescribing Convenia was given in 138 (12\%) entries; the most cited reason was an inability to orally medicate the cat in 77 (55.8\%) of these entries.

In conclusion, the majority of Convenia uses in this study were within datasheet recommendations but $26.7 \%$ were not; only $16.9 \%$ of entries had some form of microbiological evaluation recorded. The SAVSNET database was successful in providing a large source of first opinion data and will be useful for future studies on the analysis of prescribing and disease patterns in the UK.

\section{Pain relief improves sleep in dogs with osteoarthritis}

\section{David Knazovicky ${ }^{1}$, Andrea Tomas ${ }^{1}$, Alison Motsinger-Reif ${ }^{2,3}$, Duncan Lascelles ${ }^{1,2}$}

\footnotetext{
Comparative Pain Research Laboratory, Department of Clinical Sciences, College of Veterinary Medicine, North Carolina State University, Raleigh, NC, USA

Center for Comparative Medicine and Translational Research, Department of Clinical Sciences, College of Veterinary Medicine, North Carolina State University, Raleigh, NC, USA

NCSU Bioinformatics Research Center, Department of Statistics, North Carolina State University, Raleigh, NC, USA

Center for Pain Research and Innovation, UNC School of Dentistry, Chapel Hill, NC, USA
}

Chronic pain due to osteoarthritis (OA) can lead to significant disruption of sleep and increased restlessness. We hypothesized that OA in dogs is associated with a disruption of sleep. Our objective was to assess whether canine OA is associated with nighttime restlessness using objective measurement of nighttime activity and owner-assessed quality of their pet's sleep.

The study was designed as a two part prospective masked, placebo controlled study using client-owned dogs (Part A $n=60$; Part $B n=15)$. Inclusion criteria consisted of OA-associated joint pain and mobility impairment. The primary outcome measure for both parts was nighttime accelerometry. In part B, quality of sleep of the dogs was assessed using a clinical metrology instrument (Sleep and Night Time Restlessness Evaluation Score, SNORE). Part A included dogs receiving two weeks of non-steroidal anti-inflammatory drug preceded with two weeks of no treatment. Part B was a crossover study, with non-steroidal anti-inflammatory (meloxicam)/placebo administered for two weeks followed by a washout period of one week and another two weeks of the opposite treatment.

Repeated measures analysis of variance was used to assess differences between baseline and treatment in activity. Linear regression and multivariable models were used to determine variables that influenced nighttime activity. SNoRE data were evaluated using paired t-tests.

There were no significant changes in nighttime activity as a result of NSAID administration. SNORE measures indicated improvement in the quality of nighttime sleep. The SNoRE instrument detected a positive improvement due to the NSAID $(p=0.001)$, but did not detect a difference between the NSAID and placebo $(p=0.049)$ after $p$-value adjustment for multiple comparisons. There was a significant $(p<0.001)$ moderate $(r 2=0.47)$ correlation between the SNoRE and the starting CBPI pain score.

Canine OA does not appear to be associated with paininduced sleep disturbance as assessed using accelerometry, but it is clear that sleep improvement occurred with pain relief and other measured of sleep disturbance should be explored. 